\title{
Neuromuscular synapse degeneration without muscle function loss in the diaphragm of a murine model for Huntington's Disease
}

\author{
Priscila A.C. Valadão a , Matheus P.S.M. Gomes a , Bárbara C. Aragão a, \\ Hermann A. Rodrigues ${ }^{d}$, Jéssica N. Andrade a , Rubens Garcias a, \\ Julliane V. Joviano-Santos ${ }^{\text {a }}$, Murilo A. Luiz ${ }^{\text {a }}$, Wallace L. Camargo ${ }^{\text {b }}$, Lígia A. Naves ${ }^{\text {b }}$, \\ Christopher Kushmerick ${ }^{\text {b }}$, Walter L.G. Cavalcante ${ }^{c}$, Márcia Gallacci ${ }^{\text {e }}$, \\ Itamar C.G. de Jesus ${ }^{\text {b , Silvia Guatimosim }}{ }^{\text {b }}$, Cristina Guatimosim ${ }^{\text {a, ** }}$ \\ a Departamento de Morfologia, Universidade Federal de Minas Gerais, Belo Horizonte, MG, Brazil \\ ${ }^{\mathrm{b}}$ Departamento de Fisiologia e Biofísica, Universidade Federal de Minas Gerais, Belo Horizonte, MG, Brazil \\ c Departamento de Farmacologia, Universidade Federal de Minas Gerais, Belo Horizonte, MG, Brazil \\ d Departamento de Ciências Básicas da Vida, Instituto de Ciências da Vida, Universidade Federal de Juiz de Fora, Campus Governador Valadares, UFJF, GV, \\ MG, Brazil \\ e Departamento de Farmacologia, Instituto de Biociências, UNESP, Distrito de Rubião Jr., Botucatu, 18618-970 São Paulo, Brazil
}

\section{A R T I C L E I N F O}

\section{Article history:}

Received 20 October 2017

Received in revised form

27 February 2018

Accepted 8 March 2018

Available online 10 March 2018

\section{Keywords:}

Huntington's disease

BACHD

Neuromuscular junctions

Diaphragm

\begin{abstract}
A B S T R A C T
Huntington's disease (HD) is an autosomal dominant neurodegenerative disease characterized by chorea, incoordination and psychiatric and behavioral symptoms. The leading cause of death in HD patients is aspiration pneumonia, associated with respiratory dysfunction, decreased respiratory muscle strength and dysphagia. Although most of the motor symptoms are derived from alterations in the central nervous system, some might be associated with changes in the components of motor units (MU). To explore this hypothesis, we evaluated morphofunctional aspects of the diaphragm muscle in a mouse model for HD (BACHD). We showed that the axons of the phrenic nerves were not affected in 12-months-old BACHD mice, but the axon terminals that form the neuromuscular junctions (NMJs) were more fragmented in these animals in comparison with the wild-type mice. In BACHD mice, the synaptic vesicles of the diaphragm NMJs presented a decreased exocytosis rate. Quantal content and quantal size were smaller and there was less synaptic depression whereas the estimated size of the readily releasable vesicle pool was not changed. At the ultrastructure level, the diaphragm NMJs of these mice presented fewer synaptic vesicles with flattened and oval shapes, which might be associated with the reduced expression of the vesicular acetylcholine transporter protein. Furthermore, mitochondria of the diaphragm muscle presented signs of degeneration in BACHD mice. Interestingly, despite all these cellular alterations, BACHD diaphragmatic function was not compromised, suggesting a higher resistance threshold of this muscle. A putative resistance mechanism may be protecting this vital muscle. Our data contribute to expanding the current understanding of the effects of mutated huntingtin in the neuromuscular synapse and the diaphragm muscle function.
\end{abstract}

() 2018 Elsevier Ltd. All rights reserved.

\section{Introduction}

Huntington's disease (HD) is an autosomal dominant neurodegenerative disease clinically characterized by chorea,

\footnotetext{
* Corresponding author. Departamento de Morfologia- ICB, Universidade Federal de Minas Gerais, Av. Antônio Carlos, 6627, Belo Horizonte, MG 31270-901, Brazil.

E-mail address: cguati@icb.ufmg.br (C. Guatimosim).
}

incoordination, motor impersistence, psychiatric and behavioral symptoms (Finkbeiner, 2011; Walker, 2007). The worldwide prevalence of HD is 5-10 cases per 100,000 people (Baig et al., 2016). It is caused by an excessive number of CAG repeats $(\geq 37)$ in the Huntingtin gene (IT15), which translates into an elongated polyglutamine (polyQ) tail in the Huntingtin (HTT) protein (McNeil et al., 1997; Rubinsztein et al., 1996; The Huntington's Disease Collaborative Research Group, 1993).

The leading cause of death in HD patients is pneumonia (Roos, 
2010), which is mainly due to dysphagia and aspiration (Heemskerk and Roos, 2011). Respiratory dysfunction, decreased respiratory muscle strength and lung volumes, as well as swallow dysfunction, are some of the factors underlying respiratory failure and dysphagia (Heemskerk and Roos, 2011; Jones et al., 2016).

The main cause of motor symptoms is the death of striatal medium spiny and cortical pyramidal neurons (DiFiglia et al., 1995; Vonsattel and DiFiglia, 1998). Mutant HTT (mHTT) with expanded polyQ are prone to aggregate, thereby affecting several key neuronal processes such as nuclear transcription, apoptosis, mitochondrial function, axonal transport and neurotransmitter release, all eventually leading to neuronal death (Harjes and Wanker, 2003; $\mathrm{Li}$ and $\mathrm{Li}, 2004)$. Other possible explanations are: i) the mutation of the protein confers a new function to HTT that is toxic to the cell (gain of toxic function); and ii) the normal protein is sequestered in clusters formed by mHTT, thus leading to loss of normal protein function (Browne and Beal, 2004; Imarisio et al., 2008; Zuccato et al., 2010).

The HTT is a $348 \mathrm{kDa}$ multiple domain protein that is usually expressed at high levels in the central nervous system (CNS) of humans and rodents (Difiglia et al., 1997; Ferrante et al., 1997). Both the normal and mutated forms are also expressed in tissues outside the CNS such as in the skeletal muscle, heart, liver, pancreas, kidney, testis and stomach (Van Der Burg et al., 2009). Although normal HTT can be found in the cell nucleus, the protein is primarily found in the cytoplasm associated to organelles such as the Golgi complex, mitochondria, endoplasmic reticulum, synaptic vesicles, and cytoskeletal components (Hoffner et al., 2002). Interestingly, a large number of proteins interact with HTT to help axonal transport by microtubules (Schulte and Troy Littleton, 2011). Gauthier et al. (2004) showed that normal HTT is capable of improving vesicular transport by interacting with huntingtin-associated protein 1 (HAP1), which is attached to the p150 subunit of dynactin. The HAP 1 is also found associated with motor proteins such as dynein and kinesin that are proteins involved with retrograde and anterograde axonal transport (Caviston and Holzbaur, 2009; Wu and Zhou, 2009; Schulte and Troy Littleton, 2011).

Although several studies have focused on elucidating the mechanisms of neurodegeneration in the brain, the expression of the IT15 gene and HTT are not restricted to this organ, as they occur in many peripheral tissues, including skeletal muscles (Farrar et al., 2011; Sassone et al., 2009; Smith et al., 2006; Yuen et al., 2012). Previous studies have shown contractility reduction, muscle atrophy, dysregulation of contractile proteins, changes in NMJs, and electrophysiological alterations in skeletal muscles of HD mouse models (Mielcarek, 2015; Sathasivam et al., 1999). However, these studies are limited to the early stages of the disease in mice, which correspond to the pre-clinical manifestation of the disease in humans. There are a few reports considering HD in the skeletal muscles of patients with the late stages of the disease. For example. the sternomastoid muscle, a skeletal neck muscle important for head stability, was investigated by Valadão et al. (2017).

One feature of HD is the progressive weakness and uncoordinated movements of the face, neck, lips and diaphragm muscle, which underlie the chewing and swallowing difficulties that arise in patients later in their life, when the first symptoms of the disease appear (Brotherton et al., 2012). However, the structural and functional alterations in the diaphragm of HD patients remains poorly understood. We have previously described neuromuscular synaptic defects in the diaphragm of 3-months-old BACHD mouse model for HD, which corresponds to the pre-clinical stage in humans. We found morphological alterations in the shape and circumference of synaptic vesicles, decreased exocytosis and a reduction in the amplitude of miniature endplate potentials (MEPPs) in diaphragm NMJs from BACHD mice (de Aragão et al.,
2016). However, the main impairments in diaphragm function occur at later stages of the disease and virtually nothing is known about the changes in this muscle in patients with advanced HD.

To further understand the mechanisms underlying HD progression, we investigated the motor pathology of the diaphragm nervemuscle communication in the 12-months-old BACHD mouse model, which corresponds to the late clinical manifestation of the disease in humans. The BACHD mouse is a particularly attractive model because it shows symptoms that closely resemble human HD such as progressive motor and cognitive impairment, psychiatriclike disturbances and striatal neuronal loss. Moreover, it allows for the study of the later stages of the disease (Gray et al., 2008).

\section{Materials and methods}

\subsection{Drugs and chemicals}

FM1-43, FM1-43fx, $\alpha$-bungarotoxin ( $\alpha$-btx) and ProLong ${ }^{\circledR}$ Gold antifade were purchased from Invitrogen ${ }^{\mathrm{TM}}$; D-tubocurarine and ADVASEP-7 were purchased from Sigma-Aldrich. All other chemicals and reagents were of analytical grade.

\subsection{Animals and ethical procedures}

The experimental procedures were carried out in accordance with the protocol approved by the local Ethics Committee on Animal Experimentation - CEUA/UFMG - protocol 036/2013. All efforts were made to minimize animal suffering and to reduce the number of animals used. This study was not pre-registered. The experimental procedures in this work were performed at Departments of Morphology and Physiology of Universidade Federal de Minas Gerais (UFMG) and the Department of Pharmacology, Institute of Biosciences of the Universidade do Estado de São Paulo (UNESP). We used the male mouse from the strains FVB/NJ wildtype (WT) and FVB/N-Tg (HTT*97Q) IXwy/J (BACHD) (RRID: SCR $\# 008197)$. These transgenic animals were purchased from the Jackson Laboratory (Bar Harbor, ME, USA) and were housed at the animal care facility of the Department of Physiology and Biophysics at the Universidade Federal de Minas Gerais (UFMG). Upon arrival, the animals were housed at $23^{\circ} \mathrm{C}$ on a $12 \mathrm{~h}$ light $/ 12 \mathrm{~h}$ dark cycle with food (Nuvilab CR-1) and water provided ad libitum. All animals used in this study were genotyped 10 days after birth using multiplex PCR (HTT-Forward: CCGCTCAGGTTCTGCTTTTA/ HTT-Reverse: GGTCGGTGCAGCGGCTCCTC.

Actin- Forward: TGGAATCGTGTGGCATCCATCA/Actin- Reverse: AATGCCTGGGTA CATGGGGTA).

All animals used in this study were appropriately identified by numbers according to their genotype (WT or BACHD) and separated into mini-isolator cages (length: $48.3 \mathrm{~cm}$; width: $33.7 \mathrm{~cm}$; height: $21.4 \mathrm{~cm}$ ) with a maximum of four animals per cage. The mice were randomly divided into two groups using a table of random numbers. The number of experiments is provided in the figure legends/results section. The experimental groups remained constant from the beginning to the end of the study. For all experiments involving morphology and electrophysiology techniques, mice from both genotypes (WT and BACHD) were deeply anesthetized with ketamine/ xylazine $(0.1 \mathrm{~mL} / 20 \mathrm{~g})$ in accordance with the CEUA/UFMG protocol. All surgical procedures are described in the appropriate following sections. The experimental procedures were performed in the afternoon and by the end of each surgical procedure, animals were euthanized by a super-dosage of anesthetics.

\subsection{Nerve-muscle preparation}

The phrenic nerve was processed following the transmission 
electron microscopy (TEM) protocol (see below, topic 2.8). The semi-thin cross sections $(300 \mathrm{~nm})$ obtained and stained with toluidine blue as described were used to capture images of whole phrenic nerve cross-sections from WT and BACHD mice using a 20x objective in a ZEISS Axio Lab. A1 microscope. The cross-sectional area of the nerve was measured using ImageJ plugins (NIH). To quantify axonal myelination we used the G-ratio, which was calculated measuring the axonal inner diameter and dividing it by the outer diameter following the formula: $G=d / D$, where $G$ is the $\mathrm{G}$-ratio, $d$ is the inner diameter, and $D$ is the outer diameter (Chau et al., 2000).

For FM1-43 experiments, the diaphragm muscle was dissected out, split in two hemi diaphragms and bathed in mouse Ringer solution ( $135 \mathrm{mM} \mathrm{NaCl}, 5 \mathrm{mM} \mathrm{KCl}, 2 \mathrm{mM} \mathrm{CaCl}_{2}, 1 \mathrm{mM} \mathrm{MgCl}_{2}, 12 \mathrm{mM}$ $\mathrm{NaHCO}_{3}, 1 \mathrm{mM} \mathrm{Na} 2 \mathrm{HPO}_{4}$, and $11 \mathrm{mM}$ D-glucose) that was bubbled with a mixture of $95 \% \mathrm{O}_{2}-5 \% \mathrm{CO}_{2}$ and the $\mathrm{pH}$ adjusted to 7.4 .

For Transmission Electron Microscopy (TEM) experiments, the diaphragm muscles were fixed in ice-cold modified Karnovsky solution fixative (4.0\% paraformaldehyde and $2.5 \%$ glutaraldehyde in $0.1 \mathrm{M}$ sodium cacodylate buffer).

\subsection{Staining of pre and post-synaptic elements}

Experiments were performed as described previously (Rodrigues et al., 2013). Briefly, immediately after dissection, the diaphragm muscles were labeled with $\alpha$-btx- Alexa Fluor 555 $(12 \mu \mathrm{M})$ for $20 \mathrm{~min}$ to visualize the clusters of nicotinic receptors in the postsynaptic membrane. FM1-43fx $(8 \mu \mathrm{M})$ was added to stain recycling synaptic vesicles during stimulus with a high- $\mathrm{K}^{+}$solution $(60 \mathrm{mM} \mathrm{KCl})$ for $10 \mathrm{~min}$. After stimulation, the diaphragm muscle preparations were maintained at rest in normal Ringer solution with FM1-43fx for 10 min to guarantee maximal dye uptake during compensatory endocytosis. Excess of FM1-43fx adhering to the muscle plasma membrane and nerve terminal membrane was removed during a 40 min washing period in mouse Ringer solution devoid of FM1-43fx. To reduce the background fluorescence, we added Advasep-7 (1 mM) during the washing step (after FM1-43fx staining). Diaphragms were fixed with $4 \%$ paraformaldehyde for 40 min and then mounted onto slides using ProLong ${ }^{\circledR}$ Gold (Invitrogen, SP, Brazil) mounting medium.

\subsection{Confocal microscopy and image analysis}

Confocal microscopy experiments followed the steps described in previous works published by our group (Valadão et al., 2017; Rodrigues et al., 2013). Images of NMJs stained with FM1-43fx and $\alpha$-btx - Alexa Fluor 555 were acquired with a confocal laser scanning microscope (Zeiss LSM 880 - located at Center of Acquisition and Processing of Images (CAPI) - ICB/UFMG), using an oil immersion objective (63x/NA 1.4) for quantification of pre and postsynaptic elements. The excitation light came from an argon laser $(488 \mathrm{~nm})$ and the emission spectrum was set from 507 to $564 \mathrm{~nm}$ to FM 1-43fx. To visualize the $\alpha$-btx - Alexa Fluor 555 staining, we used a helium-neon laser $(543 \mathrm{~nm})$ and the emission light was collected (577-697 nm). Optical sections in Z series mode were collected at $2.0 \mu \mathrm{m}$ intervals. During image acquisition, whole hemi diaphragms were scanned and the images were obtained from muscle areas with stained pre and post-synaptic terminals. Quantitative analyses of nerve terminals were carried out with Image J software (Wayne Rasband, National Institutes of Health, USA). For each set of experiments, the images were converted to a gray scale format of 8 bits. All marked synaptic elements were individually evaluated and the mean fluorescence intensity was considered for comparisons between genotypes. The nerve terminals were identified considering their colocalization with nicotinic acetylcholine receptors ( $\mathrm{nAChR}$ ) clusters.

We used the method of particles analysis to obtain the NMJs fragmentation index based on the pixels presented in each image (Pratt et al., 2013). Briefly, the images were converted into a binary image pattern and then skeletonized. Next, to describe the connectivity for each pixel in the image, a histogram was generated using the Binary Connectivity Class plugin from Image (Pratt et al., 2013). We analyzed the degree of fragmentation in pre- and postsynaptic elements comparing muscle samples obtained from WT and BACHD mice. The parameters adopted for fragmentation were defined according to evaluation criteria that establish fragmentation by five or more islands both in the presynaptic and postsynaptic membranes, as described by Valdez et al. (2010).

\subsection{Monitoring exocytosis with FM1-43}

These experiments were performed as described in de Aragão et al. (2016) and Rodrigues et al. (2013). The fluorescent dye FM143 binds reversibly to the outer leaflet of biological membranes membrane without permeating them, being a powerful tool to track exocytosis, endocytosis and recycling of secretory granules or vesicles (Betz and Bewick, 1992). FM1-43 binds to the synaptic membrane and after a stimulus that causes exocytosis of synaptic vesicles and consequently a compensatory endocytosis, the fluorescent dye is incorporated. This results in a typical staining pattern of the synaptic vesicles (Betz and Bewick, 1992). When the nerve terminals are subjected to a new round of stimulation in the absence of FM1-43 in the external medium, the dye is released into the hydrophilic medium, leading to a decrease in the fluorescence intensity, which represents the exocytosis of synaptic vesicles (Rizzoli and Betz, 2004; Betz et al., 1996; Betz and Bewick, 1992). The neuromuscular preparations were stimulated by the addition of a saline solution containing a high concentration of potassium $(60 \mathrm{mM} \mathrm{KCl})$ in the presence of the vital dye FM1-43 $(4 \mu \mathrm{m})$ for $10 \mathrm{~min}$. After stimulation, the preparation was left for 10 min to rest in Ringer solution containing FM1-43. Then, the preparation was washed in Ringer solution devoid of FM1-43 for at least 20 min to allow the excess of labeled FM1- 43 adhered to the membrane of the synaptic terminal and the muscle cell membrane to be removed. The preparations were incubated in the presence of $\mathrm{D}$-tubocurarine $(16 \mu \mathrm{M})$ to prevent muscle contractions during the entire experimental procedure. For each animal, we used only one presynaptic terminal for monitoring the destaining process (exocytosis).

\subsection{Optical imaging and analyses}

Images of NMJs stained with FM1-43 were acquired using a fluorescence microscope (Leica DM2500) coupled to a Leica DFC 345FX camera and visualized in a microcomputer with the Leica Application Suite software (LAS). The microscope was equipped with a water immersion objective (63x, 0.95NA). Excitation light came from a 100-W Hg lamp and passed through filters (505/ $530 \mathrm{~nm}$ ) to select the fluorescence spectrum appropriate to the excitation of the FM1-43. All image adjustment variables such as binning and the exposure time were maintained constant for images acquired in the same experiment. Image analyses were performed using the software Image J allowing quantification of the brightness of several regions of interest. The mean fluorescence intensity was determined for each group of fluorescent spots and plotted as a percentage of its mean initial fluorescence using Microsoft Excel and Graph Pad Prism 4.0.

\subsection{Transmission electron microscopy}

These experiments were performed as described in Rodrigues 

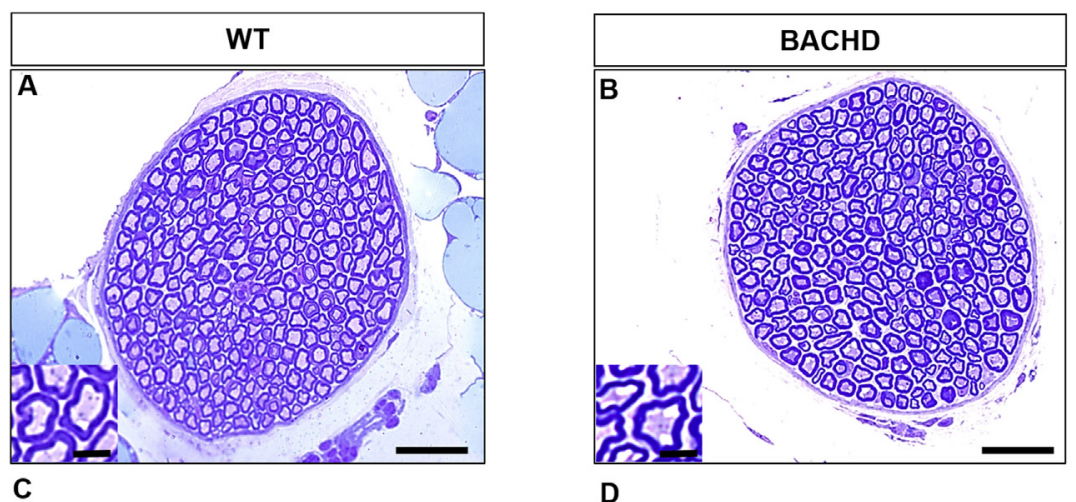

D
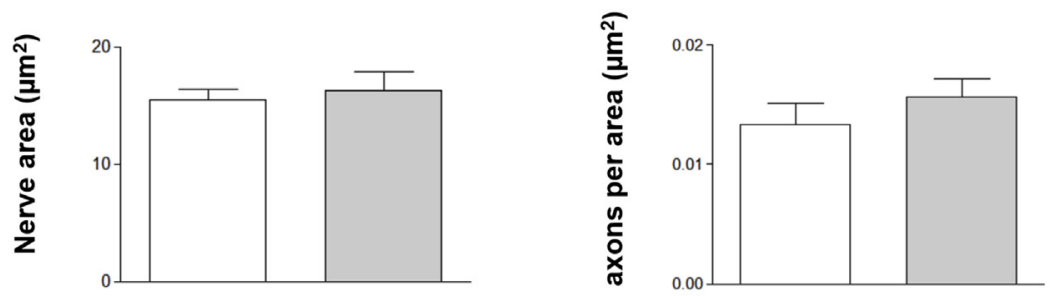

E
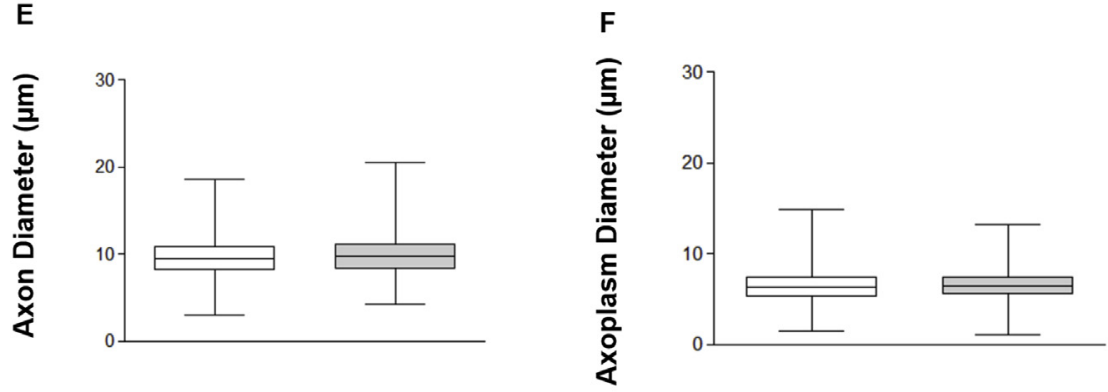

G

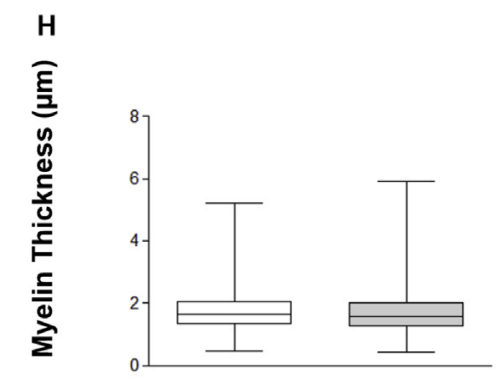

I
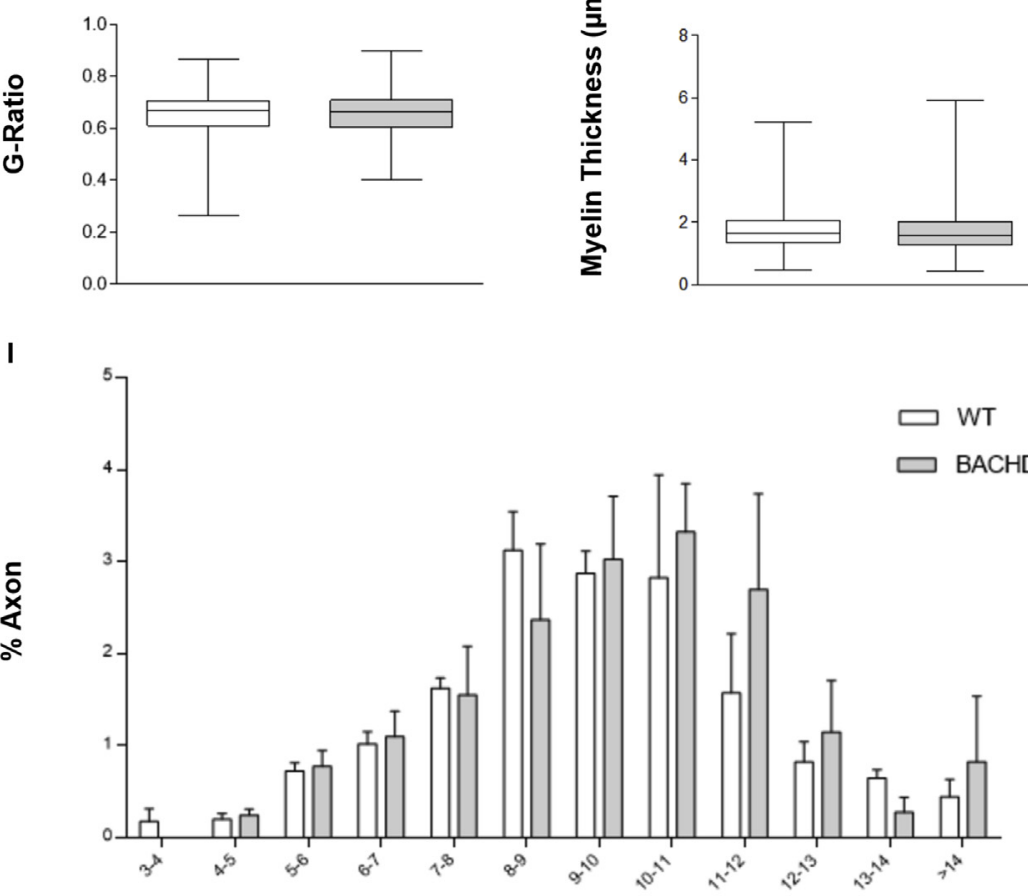

Diameter $(\mu \mathrm{m})$ 

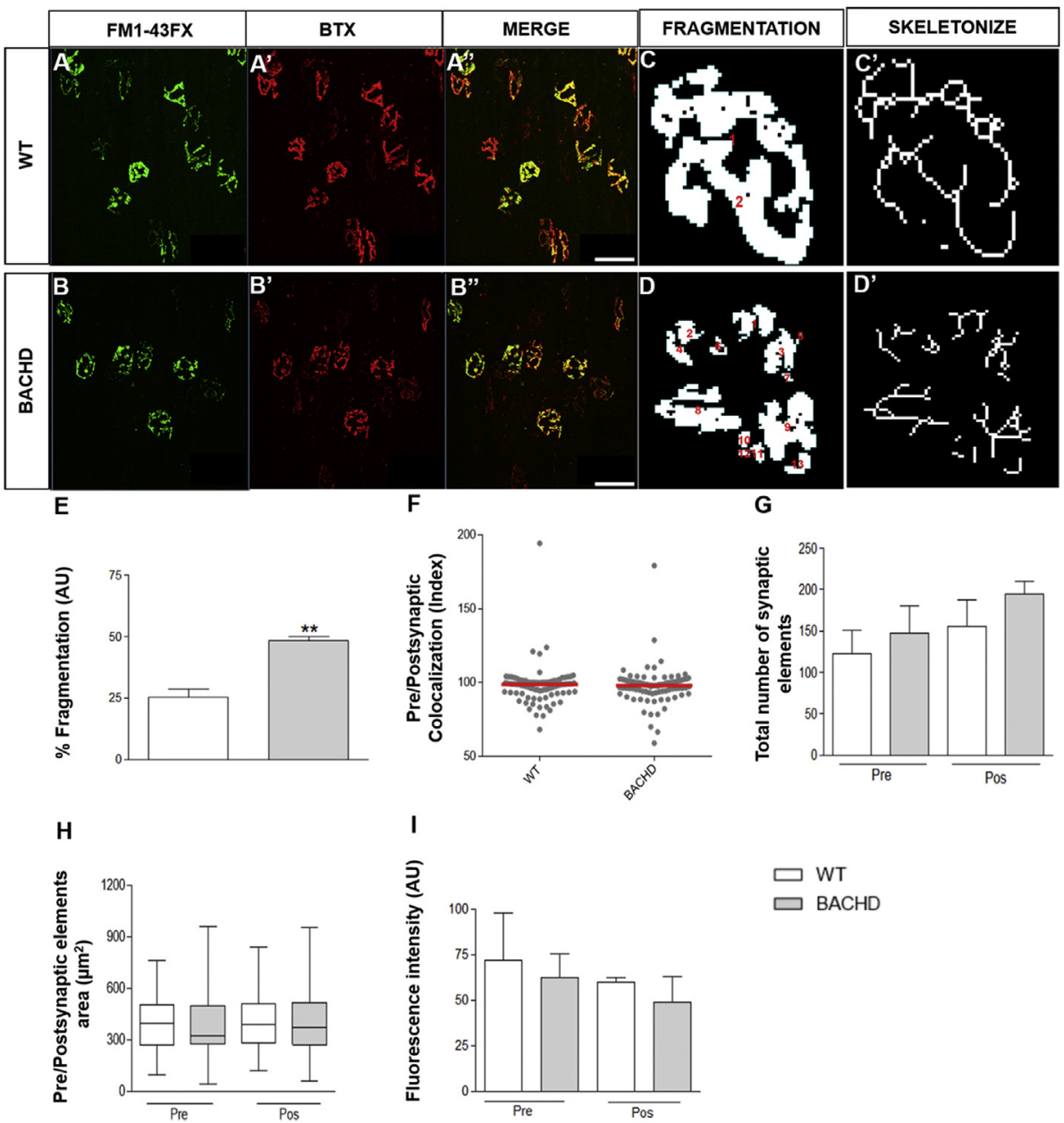

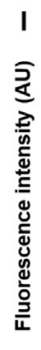

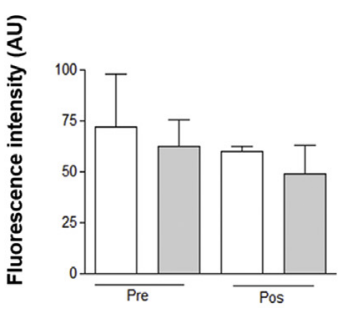

$$
\begin{aligned}
& \square \text { WT } \\
& \square \text { BACHD }
\end{aligned}
$$

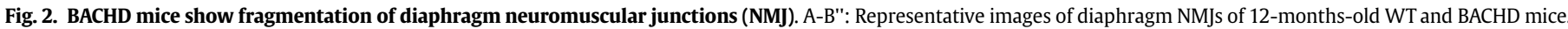

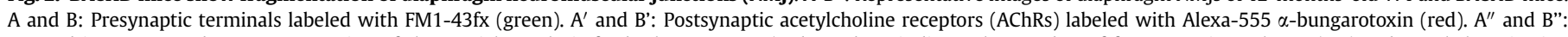

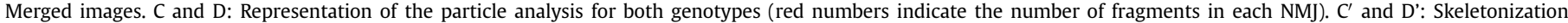

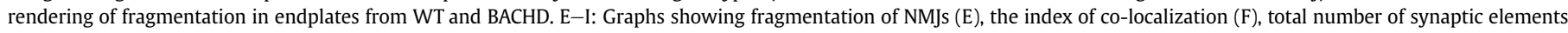

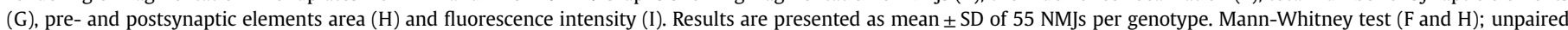

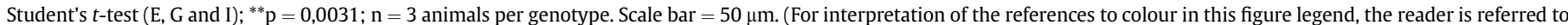
the Web version of this article.)

et al. (2013). For ultrastructural analysis, diaphragm muscles were fixed overnight in a modified Karnovsky solution at $4{ }^{\circ} \mathrm{C}$. After fixation, the samples were washed with cacodylate buffer $(0.1 \mathrm{M})$. The preparations were cut into several pieces, post-fixed in reduced osmium ( $1 \%$ osmium tetroxide containing $1.6 \%$ potassium ferrocyanide) for $90 \mathrm{~min}$ at $4{ }^{\circ} \mathrm{C}$, washed with water, contrasted en bloc with uranyl acetate ( $2 \%$ uranyl acetate in deionized water) and dehydrated through an ascending series of ethanol solutions. After dehydration, the samples were embedded in EPON resin. Then, the blocks were sectioned in semi-thin sections $(300 \mathrm{~nm})$ that were placed onto glass slides and stained with toluidine blue to aid the selection of regions of interest. Ultra-thin sections $(50 \mathrm{~nm})$ were collected on 300 mesh copper grids and contrasted with lead citrate. The sections were viewed with a Tecnai-G2- Spirit-FEI/Quanta electron microscope ( $120 \mathrm{kV}$ Philips) located at the UFMG's

\section{Microscopy Center.}

\subsection{TEM imaging analysis}

NMJs were selected based on the presence of mitochondria and junctional folds in the postsynaptic membrane. Single sections through terminals of interest were traced and the cross-section area of each nerve terminal and the number of synaptic vesicles were determined. Analyses were performed as described in Rodrigues et al. (2013).

Vesicle circumference was measured using equation $2 \pi$ [(d $2+\mathrm{d} 2$ 2)/2] 0.5 considering the longest diameter (d) and the diameter at right angles $\left(\mathrm{d}_{2}\right)$ (Van der Kloot et al., 2002). Synaptic vesicles shape was determined using the equation: shape factor $=$ $(4 \times \pi \times$ area $) /(\text { perimeter })^{2}$. This parameter reaches a maximum of

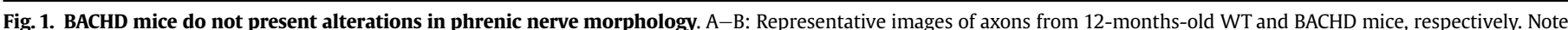

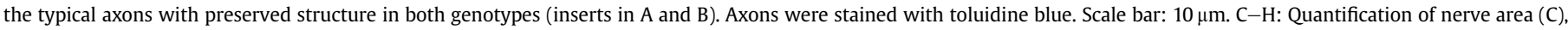

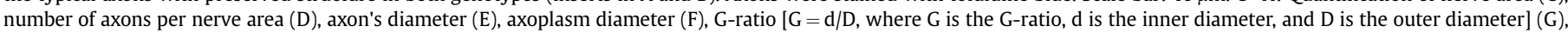

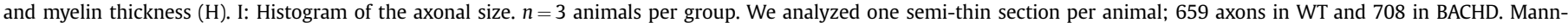

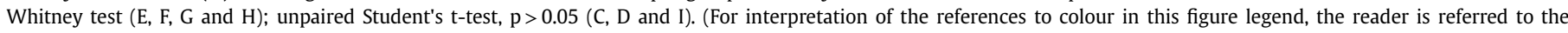
Web version of this article.) 


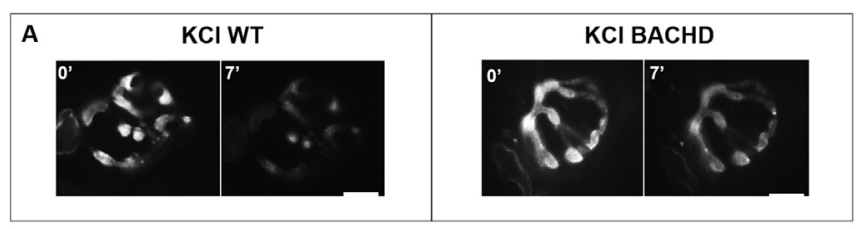

B

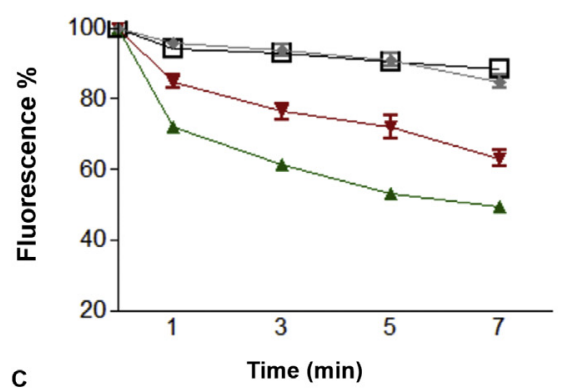

曰 Photobleaching WT

$\rightarrow$ Photobleaching BACHD

$\star \mathrm{KClWT}$

$\rightarrow \mathrm{KClBACHD}$

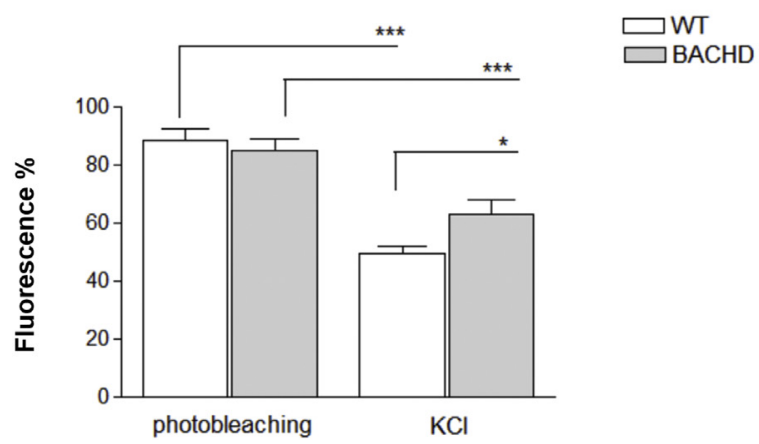

Fig. 3. BACHD mice show decreased synaptic vesicles exocytosis in the diaphragm nerve terminals. A: Representative images of the axon terminal of WT and BACHD diaphragm labeled with FM1-43 before (time $0^{\prime}$ ) and 7 min (time $7^{\prime}$ ) after the depolarizing stimulus. B: Fluorescent signal decay during $7 \mathrm{~min}$ following the depolarizing stimulus in WT (green) and BACHD (red) nerve terminals. Black and gray curves represent fluorescence decay control due to photobleaching. C: Graph showing fluorescence decay due to photobleaching and exocytosis evoked by $\mathrm{KCl}$ stimulus in WT (white) and BACHD (gray) animals. Five fluorescent points per nerve terminal were analyzed and four animals per genotype were used for quantification. The results express the mean \pm SEM of 20 fluorescent points for each experimental group. Unpaired Student's t-test; ${ }^{*} \mathrm{p}<0.05,{ }^{* * *} \mathrm{p}<0.0001$. Scale bar $=10 \mu \mathrm{m}$. (For interpretation of the references to colour in this figure legend, the reader is referred to the Web version of this article.)

1 for a circular object (Croft et al., 2005). The values for circumference and shape factor are shown by a histogram and a cumulative probability plot and compared using the Mann Whitney test. The graphs were plotted using the software Minitab and Sigma Plot 10.00 (SyStat Software) Graph Pad Prism 4.0. While determining the average circumference of the synaptic vesicles, we did not correct for variability in the plane of the section through which the vesicles were cut. Although this is likely to underestimate vesicle diameter and lead to variability in size and shape, we did not expect this to change the interpretation of our results. Indeed, these effects should be similar for the identically prepared samples from WT and BACHD mice. Van der Kloot et al. (2002) used a similar approach.

\subsection{Electrophysiology}

Hemi-diaphragm nerve-muscle preparations were prepared as described above and maintained in Ringer solution bubbled with carboxygen (5\%CO2/95\% O2) at room temperature (22-24C). For recordings, muscles were pinned onto a bed of silicon rubber (Sylgard; DowCorning, Midland, MI) in a $5 \mathrm{~mL}$ chamber on the stage of a dissecting microscope and perfused with carboxygenated Ringer at $1-2 \mathrm{~mL} / \mathrm{min}$. Microelectrodes were fabricated from borosilicate glass using a Narishige puller $(\mathrm{PN}-30)$ and had resistances of 8-15 $\mathrm{M} \Omega$ when filled with $3 \mathrm{M} \mathrm{KCl}$. Recordings were made with an Axoclamp 2A 10-X preamplifier (Molecular Devices) connected to a CyberAmp 380 (Molecular Devices) that filtered the signal at a band with of $0.1-5 \mathrm{kHz}$ and then applied further amplification by 20-100 times, as needed. A second channel recorded the DCcoupled 10 times Vm signal which was used to correct for resting potential and non-linear summation (see below). Data was sampled at $100 \mathrm{kHz}$ by a digital-to-analog converter (National Instruments) in a PC computer controlled by the program WinEDR (kindly provided by John Dempster, University of Strathclyde).

Microelectrodes were inserted into the muscle fibers in the endplate region as verified by the presence of miniature endplate potential (MEPP) with rise time $<1 \mathrm{~ms}$. To measure quantal size, tetrodotoxin $(100 \mathrm{nM})$ was included in some experiments to avoid action potentials and movement artifacts. In experiments with nerve stimulation, we used the cut-fiber method (Barstad and Lilleheil, 1968) which depolarizes the nerve fiber thus preventing muscle contraction. For stimulation, the nerve was drawn into a suction electrode and suprathreshold $0.1 \mathrm{~ms}$ voltage pulses were applied. Amplitudes of EPPs and MEPPs were corrected to a standard resting potential of $-70 \mathrm{mV}$ for intact fibers or $-36 \mathrm{mV}$ for cut fiber experiments. EPP amplitudes were further corrected for nonlinear summation (McLachlan and Martin, 1981).

To measure quantal size in intact fibers, we sampled $>100$ MEPPS from each of 5 fibers from 5 animals of each genotype. For measurements of EPP, quantal content, tetanic depression and readily-releasable pool, measurements were from 4 to 7 fibers per animal from 3 different animals per genotype. The nerve stimulation protocol consisted of 90 stimuli applied at $0.3 \mathrm{~Hz}$ followed by 1500 stimuli at $30 \mathrm{~Hz}$. MEPP amplitudes were captured during the intervals between stimuli. Quantal content was calculated by the direct method and expressed in units of EPP/MEPP, for which quantal size (i.e. MEPP amplitude) was determined separately for each fiber. Synaptic depression was calculated as the average of 30 EPP amplitudes at the end of a $50 \mathrm{~s}$ train at $30 \mathrm{~Hz}$, normalized to the amplitude of the first EPP in the train. Readily-releasable pool was estimated by fitting a line to the relationship of QC vs $\Sigma Q C$ (Elmqvist and Quastel, 1965). For these calculations, the linear fit started at the second EPP, to allow for facilitation and 10 EPPs were used for the fit. Estimates of RRP are expressed in units of EPP/MEPP (i.e. quanta).

\subsection{Neuromuscular preparation and recording of muscle contractions}

The phrenic nerve-diaphragm muscle preparation was removed and mounted for myographic recording "in vitro", as described by Gallacci and Oliveira (1994). Briefly, the preparation was mounted vertically in a conventional isolated organ-bath chamber containing $15 \mathrm{~mL}$ of Ringer's solution. This solution was gassed with $\mathrm{O}_{2}$ $(95 \%)+\mathrm{CO}_{2}(5 \%)$ and maintained at $35^{\circ} \mathrm{C}$. Muscle contractions were recorded by isometric force transducer (FT03, Grass) coupled to an AcquireLab Data Acquisition System (Gould). Indirect contractions were evoked by supramaximal strength pulses delivered from an electronic stimulator (S88K, Grass) and applied to the phrenic nerve using a suction electrode. Once mounted in the test apparatus, muscles were allowed to stabilize for $30 \mathrm{~min}$, and an indirect stimulation $(0.2 \mathrm{~Hz}, 5 \mathrm{~ms})$ was used to establish the muscle viability. 
A
ปั

告

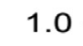

0.8

$0.6-$

$0.2-$

$0.0-$

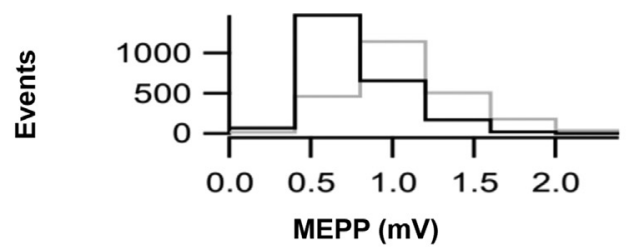

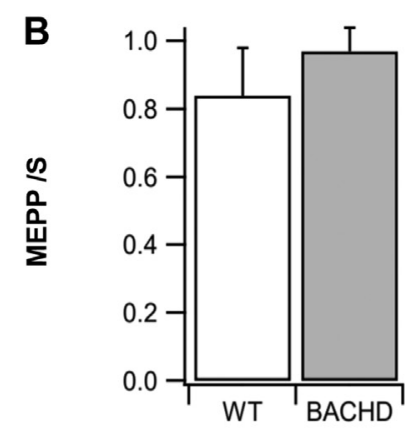

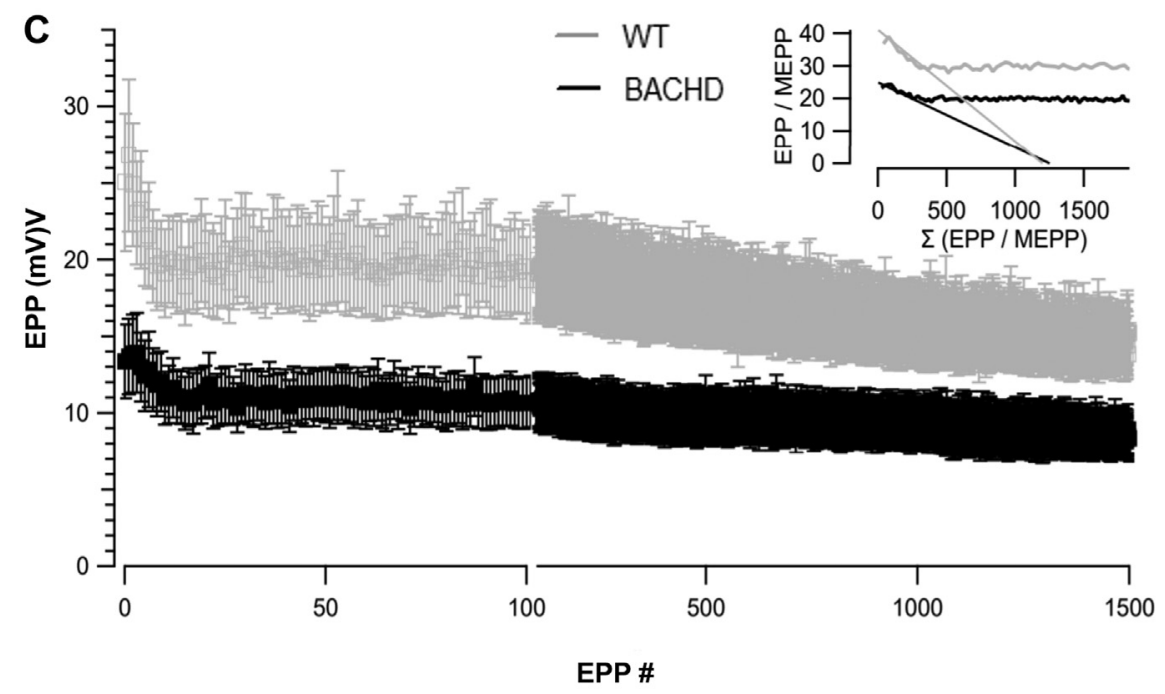

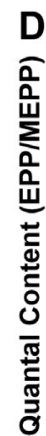

$\mathbf{E}$

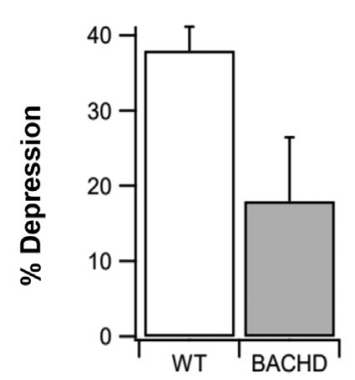

$\mathbf{F}$

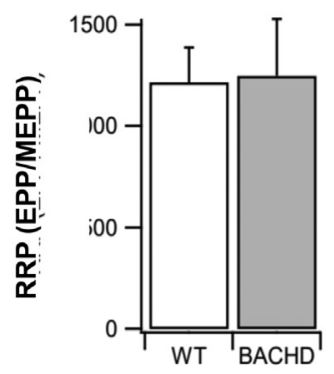

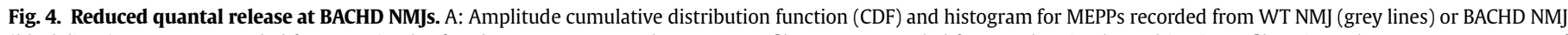

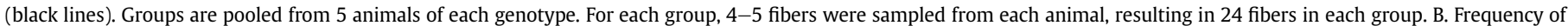

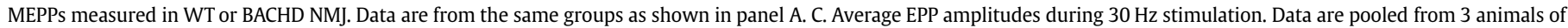

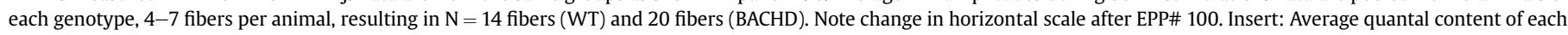

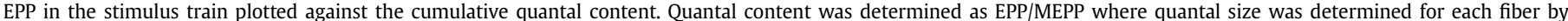

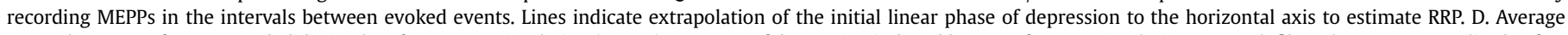

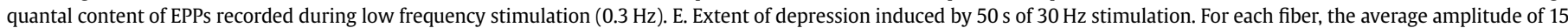

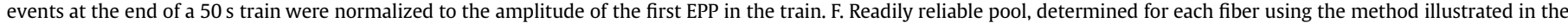

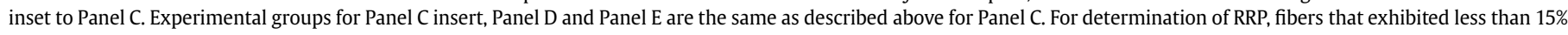
depression generated unreliable estimates and were excluded from analysis, resulting in $\mathrm{N}=12$ fibers for WT and 14 fibers for BACHD.

\subsection{Safety margin of synaptic transmission}

The overall safety margin of the synaptic transmission was evaluated from the susceptibility of indirectly evoked contractions of the diaphragm muscle to the blockade induced by the addition of increasing concentrations of pancuronium bromide or magnesium to the organ bath, allowing $10 \mathrm{~min}$ between each concentration. The ratio of muscle tension in the presence and absence of pancuronium or magnesium was used to estimate the safety margin of neuromuscular transmission.

Other preparations were submitted to a gradual increase of tetanic stimulation, starting at $70 \mathrm{~Hz}$ and ending at $220 \mathrm{~Hz}$, with a gradual increase of $10 \mathrm{~Hz}$. Each tetanus lasted $5 \mathrm{~s}$ with an interval of 10 min between them. During this interval, supramaximal pulses $(0.2 \mathrm{~Hz} ; 0.5 \mathrm{~ms})$ evoked indirect contractions. The ability of the preparation to support the different tetanus was assessed by the 

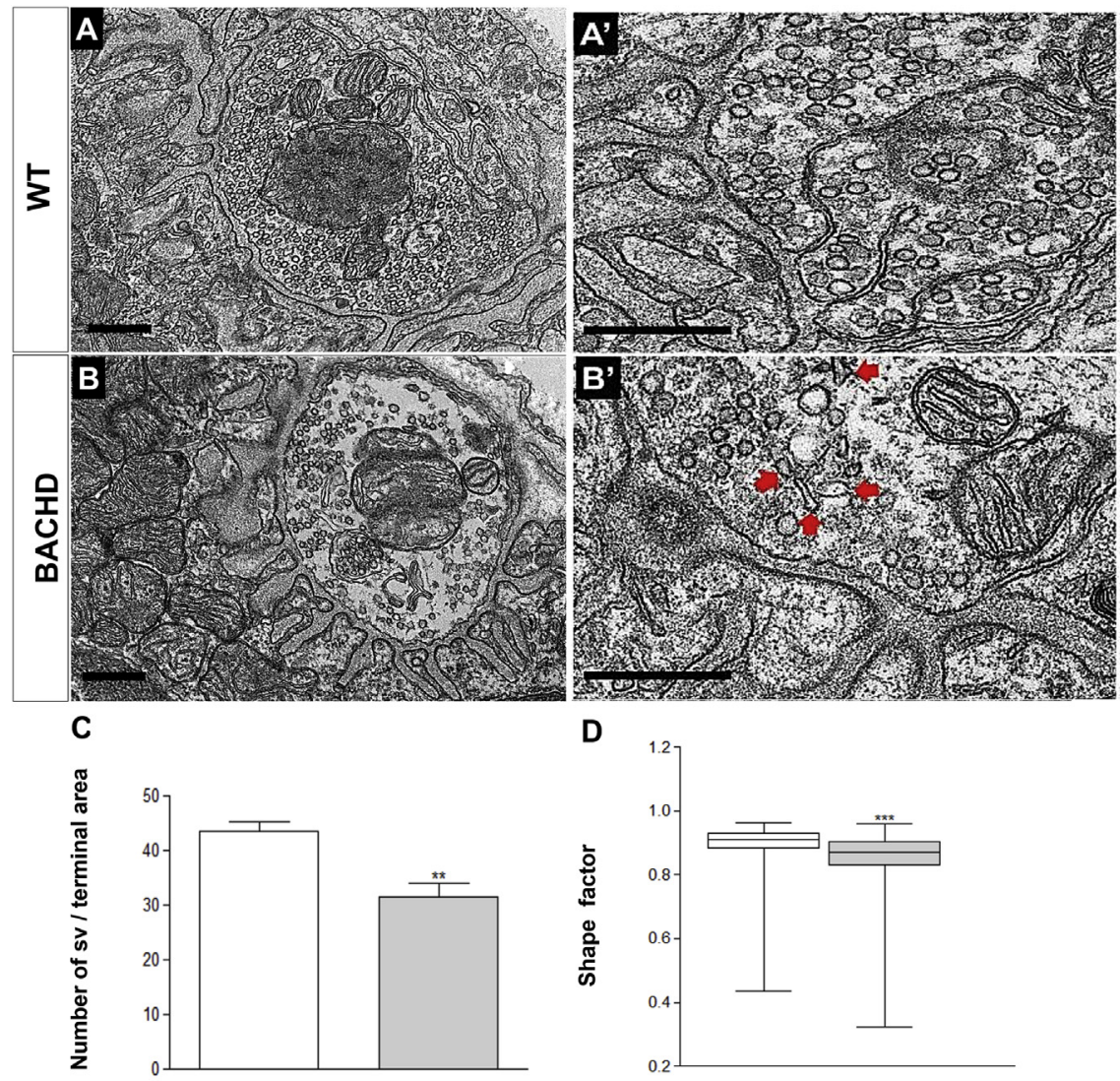

E

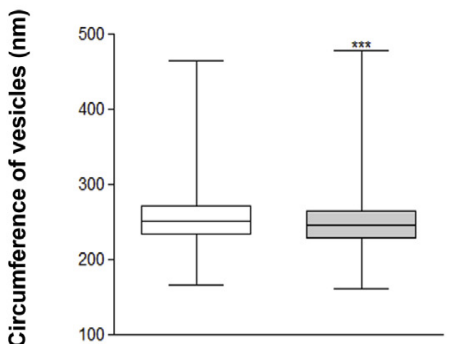

$\mathbf{G}$

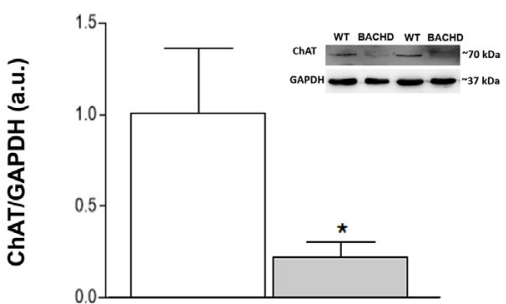

$\mathbf{F}$

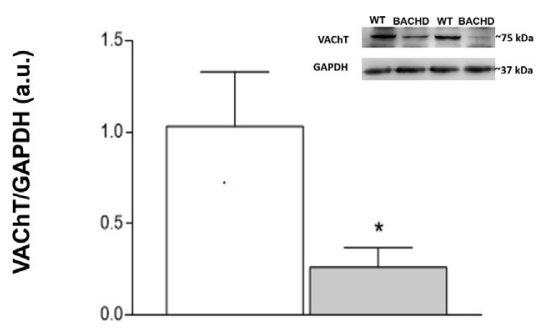

H

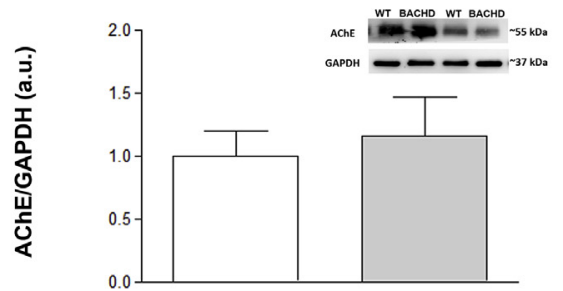

Fig. 5. BACHD mice present ultrastructural changes in the morphology of the synaptic vesicles of diaphragm NMJs. A: Representative image of a WT diaphragm nerve terminal. B: Representative images of a BACHD nerve terminal presenting synaptic vesicles with heterogeneous sizes. Scale bar $=500 \mathrm{~nm}$. $\mathrm{A}^{\prime}$ and B': Representative images showing nerve terminals with changes in the size and shape of the synaptic vesicles (red arrows). Scale bar $=500 \mathrm{~nm}$. C: Graph representing the number of synaptic vesicles per terminal area (Unpaired student's t-test; ${ }^{*} \mathrm{p}=0.0286$ ). D: Boxplot comparing the synaptic vesicles shape in WT and BACHD mice (Mann Whitney test, ${ }^{* * *} \mathrm{p}<0.0001$ ). E: Boxplot comparing synaptic vesicles circumference in WT and BACHD mice (Mann Whitney test, ${ }^{* * *} \mathrm{p}<0.0001$ ). We analyzed at least 15 NMJs profiles from three animals per genotype. All integrally visualized synaptic vesicles were used for the quantification of shape and circumference. A total of 3723 synaptic vesicles were analyzed for WT and 2343 for BACHD F-H: Western blot analysis of VAChT (F), ChAT (G) and AChE (H) proteins in diaphragm muscle of WT and BACHD. GAPDH was used as a control of protein loading between experiments. GAPDH protein levels are similar between WT and BACHD. Data are presented as a percentage of WT (Unpaired student's t-test; ${ }^{* * *} \mathrm{p}=0.0286,{ }^{*} \mathrm{p}=0.04$ ). (For interpretation of the references to colour in this figure legend, the reader is referred to the Web version of this article.) 
relationship between the amplitude of the initial tetanic peak amplitude and the moment at which the stimulus was ended.

\subsection{Fatigue resistance}

After the diaphragm muscle was stabilized, the preparations were submitted to a repeat sequence of 30 tetanic contractions, with $5 \mathrm{~s}$ long, $130 \mathrm{~Hz}$ and an interval of $20 \mathrm{~s}$. Fatigue resistance was defined as the amplitude of the last contraction of the series relative to the first, expressed as a percentage. Results were expressed as mean \pm S.E. Data were analyzed by ANOVA complemented by the Tukey-Kramer test. Values of $\mathrm{p}<0.05$ were considered significant.

\subsection{Western blot}

To detect the vesicular ACh transporter (VAChT), Choline acetyltransferase (ChAT) and Acetylcholinesterase (AChE) in diaphragm muscles, $50 \mu \mathrm{g}$ of protein were separated by SDS-PAGE. The antibodies used and their sources are as follow: anti-VAChT (1:2000; Sigma-Aldrich Cat\# V5387 RRID: AB_261875), anti-ChAT (1:1000; Abcam Cat\#ab70219 RRID: AB_1209541) and anti-AChE (1:1000; Thermo Fisher Scientific Cat\#MA3-042 RRID: AB_325478) Glyceraldehyde-3-phosphate dehydrogenase (GAPDH; 1:3000; Santa Cruz Biotechnology Cat\#sc- 20356 RRID: AB_641103). Validation data for each antibody were obtained from the data-sheet provided by the company. Immunodetection was carried out using enhanced chemiluminescence (Amersham Biosciences). Protein levels were expressed as a ratio of optical densities. GAPDH was used as a protein loading control.

\subsection{Statistical analysis}

All data were registered using Microsoft Excel and were plotted using the program GRAPHPAD PRISM 6. For data with normal distribution, values were represented as the mean \pm standard deviation (mean \pm SD) and statistical significance was evaluated using the unpaired Student's t-test. When data were not normally distributed, the values were represented as the median and the Man-Whitney test was used to assess statistical significance. Values of $\mathrm{p}<0.05$ were considered significant. Exact $\mathrm{p}$-values are provided in the figure legends. During the analyses, the investigators were blinded to the genotypes and experimental groups. Each genotyped animal was assigned a number that was revealed to the investigator only after the analyses ended.

In this work, we used a minimum of three animals per genotype for each data set to detect a difference at $95 \%$ confidence $(a=0.05)$ and 0.8 statistical power. The exact $n$ for each experimental procedure is described in the figure legends. All data points were treated as outliers and excluded from the data analysis outside the $95 \%$ confidence interval.

\section{Results}

We previously described neuromuscular synaptic defects in the diaphragm of adult (3-months-old) BACHD mice (de Aragão et al., 2016). Herein, we asked if aging would exacerbate those defects. We initially looked at the structure of the phrenic nerve that is responsible for innervation of the diaphragm muscle in 12-monthsold BACHD and WT mice. Histological analysis of this nerve showed that, in both genotypes, the phrenic nerve was preserved (Fig. 1A-B), with no alterations in the analyzed parameters: i) nerve area (C) ii) number of axons per area (D); iii) axon diameter (E); iv) axoplasm diameter (F); v) G-ratio (G); and vi) size of the axons $(\mathrm{H})$.

Labeling the nerve-muscle preparations with the activity- dependent fluorescent marker FM1-43fx and the postsynaptic nicotinic receptors for ACh with the $\alpha$-btx conjugated to Alexa Fluor 555 , revealed significant alterations in the motor nerve terminals during depolarizing stimulus. Fig. 2 shows representative images of pre- and postsynaptic elements of the diaphragm NMJs of WT (Fig. 2A-A") and BACHD (Fig. 2B- B") mice. Fragmentation analysis showed that diaphragm NMJs from 12-month-old BACHD mice are more fragmented than diaphragm NMJs of WT animals (Fig. $2 \mathrm{C}-\mathrm{C}^{\prime}$, D-D' and E). The quantitative analysis of the pre- and postsynaptic elements showed no statistically significant differences in the following morphological parameters: i) co-localization of pre- and postsynaptic elements (Fig. 2F); ii) total number of elements (Fig. 2G); iii) area of the elements (Fig. 2H). Measuring endocytic activity through fluorescence intensity of the presynaptic elements did not reveal significant differences between the genotypes (Fig. 2I). Next, using FM1-43, we measured the exocytosis of synaptic vesicles in the diaphragm NMJs of BACHD and WT animals. The fluorescence intensity was reduced in the diaphragm NMJs of both BACHD and WT mice, especially in the later (Fig. 3B). The KClevoked decrease in the fluorescent signal in the nerve terminals was greater in the WT in comparison with the BACHD mice (Fig. 3C). Diaphragm NMJs of WT mice showed a decrease in fluorescence of approximately 50\% whereas the diaphragm NMJs of BACHD mice lost about 35\% of their fluorescent signal (Fig. 3C). No significant differences were observed between WT and BACHD photobleaching values (Fig. 3C).

Electrophysiological recordings show that MEPPs of BACHD mice have smaller amplitudes when compared with WT (Fig. 4A). In contrast, there was no significant difference in MEPPs frequency between the two genotypes. (Fig. 4B).

Since we found that BACHD animals have a decreased rate of destaining, which suggests an imparment in the exocytosis, we measured evoked release during low frequency $(0.3 \mathrm{~Hz})$ and high frequency $(30 \mathrm{~Hz})$ stimulation. In between evoked events, we captured MEPPs to calculate quantal content by the direct method (EPP/MEPP). Both EPP amplitude and quantum content were significantly reduced in BACHD animals (WT: EPP $=26.6 \pm 5.5 \mathrm{mV}$, $\mathrm{QC}=39 \pm 4.8 ; \quad$ BACHD: $\quad \mathrm{EPP}=14.0 \pm 2.5 \mathrm{mV} \quad \mathrm{QC}=24.7 \pm 3.1$, $\mathrm{p}<0.05)$. During high frequency stimulation, average EPP amplitude declined in both WT and BACHD groups, however tetanic depression was stronger in WT groups (Fig. 4E). At the end of a $50 \mathrm{~s}$ train at $30 \mathrm{~Hz}$, EPP amplitudes in WT fibers had declined by $38 \pm 3.2 \%$ of compared to a decline of only $18 \pm 8.5 \%$ in the BACHD Group ( $\mathrm{p}<0.05)$.

We estimated the size of the Releasable pool using a calculation based on the method of Elmqvist and Quastel (1965). EPPs were measured during $30 \mathrm{~Hz}$ stimulation and the QC of each EPP in the train plotted as a function of the cumulative release (Fig. 4C, insert) and a line fit to the initial, linear phase of depression and extrapolating to the horizontal axis. These data indicate an available pool of about 1200 quanta, with no significant difference between WT and BACHD animals. Based on these results, we conclude that, although the pool of vesicles available is similar in WT and BACHD $\mathrm{NMJ}$, the BACHD group have a lower release probability, resulting in fewer quantal released. These findings explain the reduction in FM143 destaining as described above. A consequence of the reduced release probability is that BACHD exhibit less synaptic depression during $30 \mathrm{~Hz}$ stimulation.

Since we observed a decrease of quantal size in the diaphragm NMJs of BACHD, we investigated whether there were ultrastructural changes in the size and shape of the synaptic vesicles in the diaphragm NMJs of these mice. The qualitative analysis of the electron micrographs of the diaphragm NMJs showed that the nerve terminals of both genotypes presented similar morphology of the terminal area and post-junctional folds (Fig. 5A-B). We also 

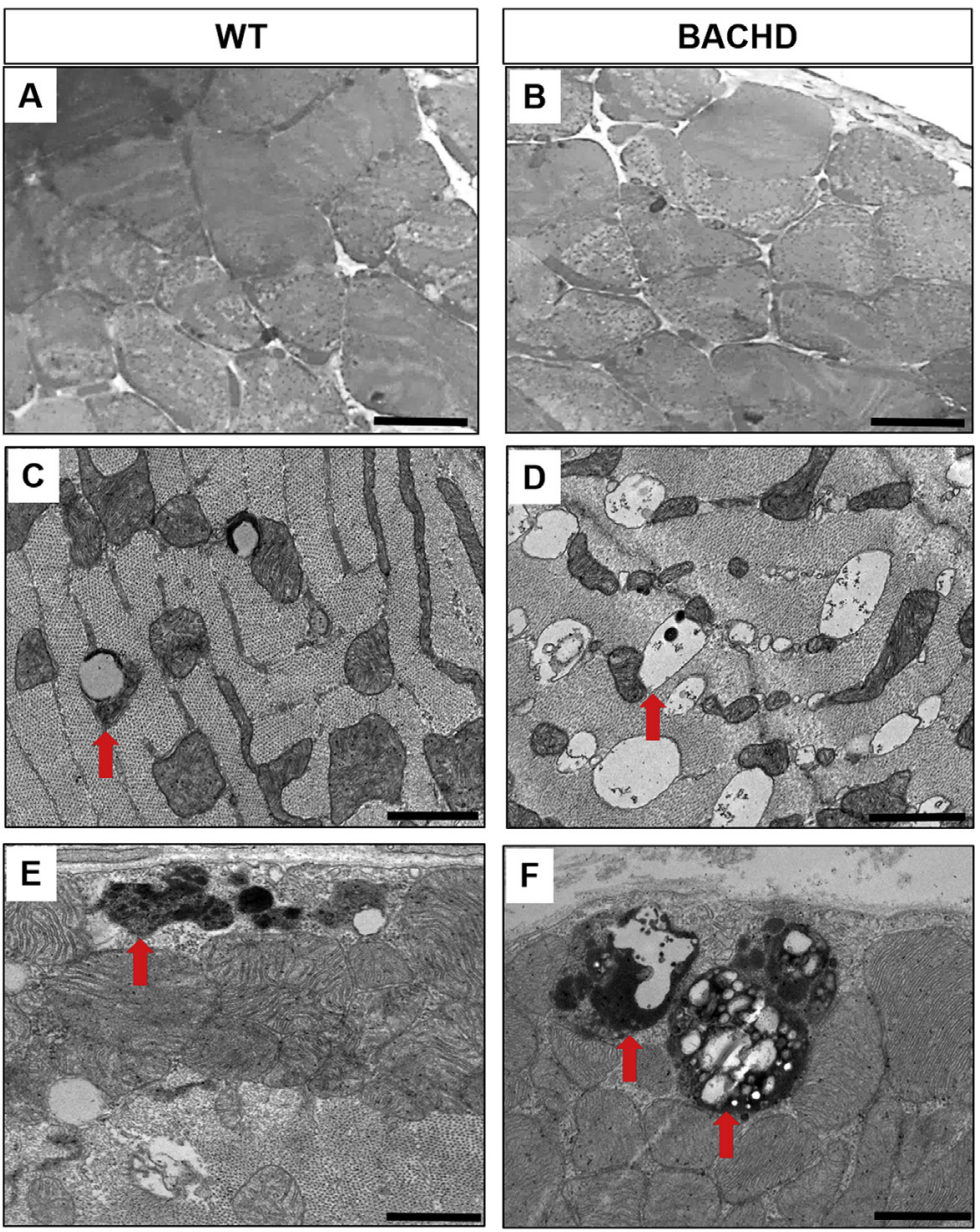

G

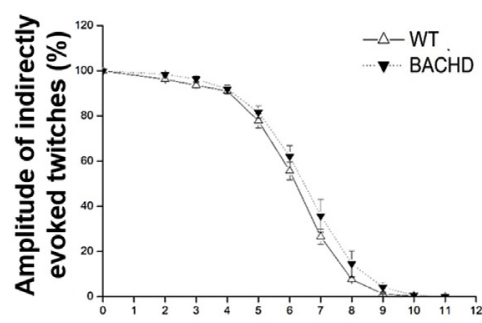

Pancuronium (x10-7 M)

I

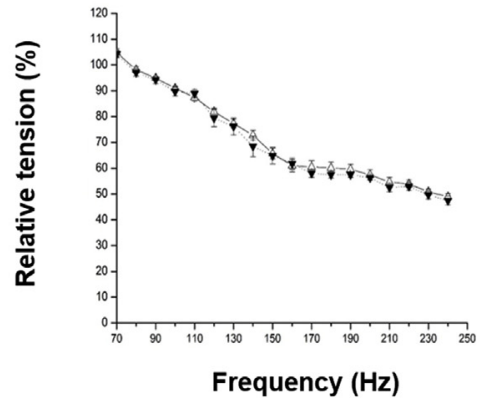

H

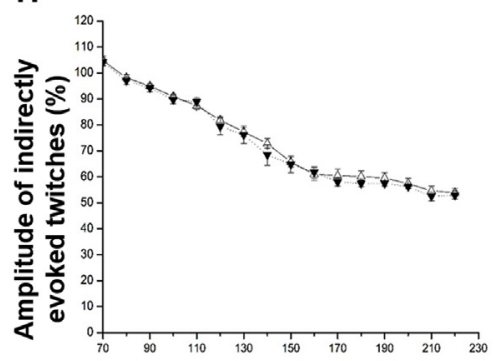

Magnesium concentration (mM)

J

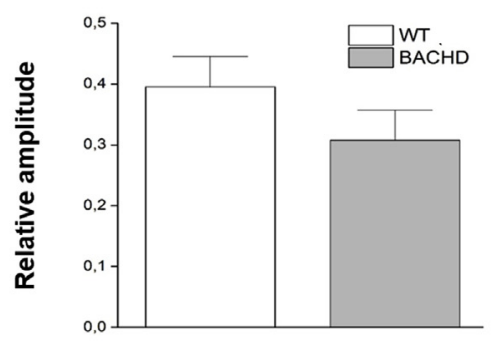

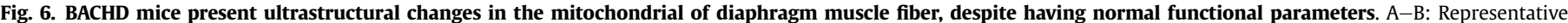

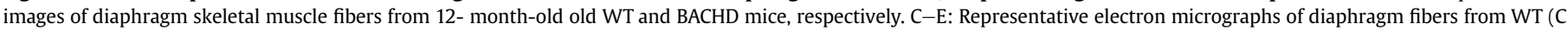

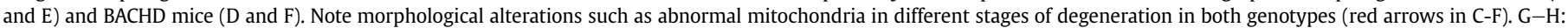

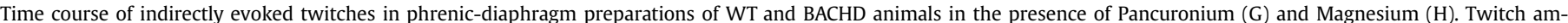

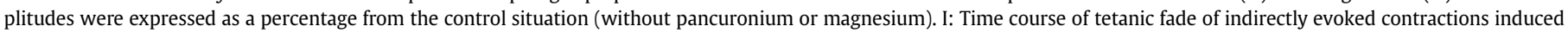


observed that the nerve terminals of BACHD animals contained less synaptic vesicles in their interior (Fig. 5 B'-C). Moreover, the diaphragm NMJs of BACHD mice presented vesicles with irregular morphology (varying sizes, flattened or with elliptical shape) (Fig. 5D). Additionally, the circumference and shape of the synaptic vesicles of the diaphragm NMJs of BACHD animals were smaller than that found in the diaphragm NMJs of WT mice (Fig. 5E). Given that the expression of cholinergic neuronal markers such as ChAT, AChE and VAChT is reduced in transgenic mice for HD (Aquilonius et al., 1975; Smith et al., 2006; Massouh et al., 2008), we also examined VAChT, ChAT and AChE protein expression levels in the diaphragm NMJs of 12-months-old BACHD animals. We found a significant reduction in the expression of VAChT and ChAT, but AChE was not reduced in the diaphragm NMJs of BACHD compared to WT mice (Fig. 5F-H).

In light of the above-reported observations, we investigated the general morphology of the diaphragm muscle fibers. The qualitative analysis showed no morphological changes in the diaphragm muscle fibers of both genotypes (Fig. 6A-B). However, ultrastructural analysis revealed abnormal mitochondria with vacuoles in the diaphragm NMJs of WT and BACHD animals, as shown in the representative images displayed in Figs. 6C-F. Lastly, we asked if all the presynaptic and muscle alterations described above could compromise diaphragm function in BACHD mice. We found no differences between the genotypes with regards to the susceptibility of neuromuscular blockade induced by pancuronium bromide (Fig. 6G) or excess of magnesium (Fig. 6H), indicating that the safety margin of diaphragm's synaptic transmission is preserved in 12-months-old BACHD mice. In addition, experiments with increasing frequencies of tetanic stimulation also showed no differences between the experimental groups (Fig. 6I). The fatigue resistance test revealed similarities between WT and BACHD animals (Fig. 6J). These data indicate that despite all morphological changes, the diaphragm muscle maintains its functional capacity in the BACHD mice.

\section{Discussion}

One of the main pathological features of HD is brain neurodegeneration, although cellular dysfunctions have also been reported in other structures such as the spinal cord motor neurons, nerves, NMJs and the skeletal muscles (Valadão et al., 2017; Mielcarek, 2015; Zielonka et al., 2014; Ribchester et al., 2004). Previous work from our group showed that 3-months-old BACHD mice exhibit: i) decreased number of synaptic elements in diaphragm NMJs; ii) reduced synaptic vesicle exocytosis; iii) changes in the form and sizes of the synaptic vesicles; and iv) decreased spontaneous ACh release, thus suggesting a deficit of neuromuscular function (de Aragão et al., 2016). To better understand the progression mechanisms of $\mathrm{HD}$, we investigated the advance of motor pathology in the diaphragm of 12-months-old BACHD mice.

In BACHD, the NMJs from phrenic nerve, which innervates the diaphragm muscle, did not differ from the WT mice regarding the index of co-localization, the total number and area of pre- and postsynaptic elements and the endocytosis process. However, diaphragm NMJs of BACHD were more fragmented compared with WT, indicating that diaphragm NMJs of BACHD mice are undergoing a neurodegenerative process. These results are in agreement with previous morphological evaluations showing an increase of NMJ fragmentations of different models for neurodegenerative diseases such as HD and ALS (Valadão et al., 2017; Valdez et al., 2010, 2012).

The decreased FM1-43 destaining rate and smaller synaptic depression observed in BACHD mice argue in favor of a decreased vesicular exocytosis rate. This indicates that middle-aged (12months-old) BACHD present alterations in exocytosis of synaptic vesicles similar to previous observations in younger animals (3month-old BACHD) (de Aragão et al., 2016). Abnormal interactions between mHTT and proteins that are involved with the exocytosis process may influence the release of neurotransmitters (Sari, 2011; Saudou and Humbert, 2016; Zuccato et al., 2010). Also, several studies demonstrated the reduction of synaptic proteins involved in synaptic vesicle exocytosis in the postmortem brain of WT animals and in HD mice. These proteins include complexin II, VSNARE (synaptobrevin 2), and Rab3A (Morton and Edwardson, 2001; Smith et al., 2005, 2007). These reports show that mHTT interacts with key proteins of the exocytosis process of synaptic vesicles, which may explain the lower rate of exocytosis seen in the BACHD in relation to WT mice.

Diaphragm NMJs of BACHD mice had a reduced quantal size, when compared with WT animals. Reduction in MEPPs' size in the 3-months-old BACHD mice has been reported by de Aragão et al. (2016), indicating that the deficit in the quantal size in BACHD mice remains at more advanced ages. This could be due to less ACh per vesicle since we observed here a decrease in ChAT and VAChT expression levels in BACHD diaphragms.

We observed no differences in the frequency of MEPPs between BACHD and WT mice, showing similarity in the rate of spontaneous release in both genotypes. On the other hand, we observed less EPPs depression. Data from the literature regarding neurotransmission are divergent, with two reports showing increased synaptic vesicles exocytosis and neurotransmitter release (Rozas et al., 2010, 2011), decreased release (Khedraki et al., 2017; and a report showing no differences in the neurotransmission mechanism (Ribchester et al., 2004). The differences between some of these studies and our observations may be due to the different transgenic models used.

We found changes in the morphological aspect of synaptic vesicles. Electron micrographs revealed that the presynaptic terminals of the diaphragm NMJs of BACHD mice possessed altered synaptic vesicles shape (elliptic and flattened) and circumference compared with the WT counterparts. de Aragão et al. (2016), also observed a similar result in 3-months-old BACHD. The mHTT binds more strongly to the synaptic vesicles compared with the normal HTT, thus altering the structure of the vesicle and affecting neurotransmitter release (Li et al., 2003), which could explain the alterations observed in the structure of the synaptic vesicles.

Reduction in VAChT protein expression can lead to reduced vesicular content of $A C h$, which can be seen as changes in both MEPPs and synaptic vesicle size in KD VAChT mice (Rodrigues et al., 2013). The reduction in quantal size we observed in the present study could be due to smaller vesicular content of ACh due to less VAChT to fill the vesicles. Moreover, transgenic HTT mice have reduced expression of cholinergic neuronal markers such as ChAT (Aquilonius et al., 1975; Massouh et al., 2008), AChE, and VAChT (Smith et al., 2006). These findings may also be related to smaller quantal size we observed. Considering that the synaptic vesicles morphology seems to correlate with the level of their filling with neurotransmitters (Budzinski et al., 2009; Rodrigues et al., 2013; Van der Kloot et al., 2002), we suggest that the changes in the

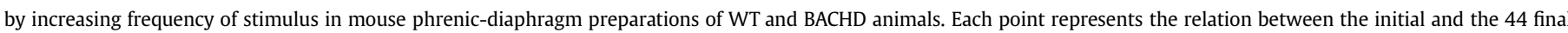

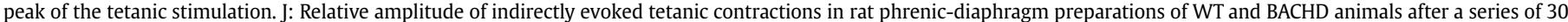

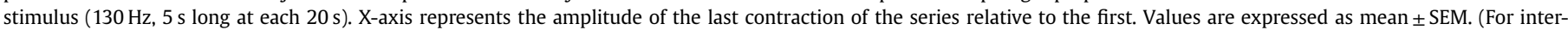
pretation of the references to colour in this figure legend, the reader is referred to the Web version of this article.) 
circumference of the synaptic vesicles described here may be a consequence of decreased filling with ACh. Further research will be needed to verify this hypothesis.

Ultrastructural changes were observed in the diaphragm muscle of 12-months-old BACHD animals, including mitochondria at different stages of degeneration. However, these changes were also found in the control muscles. According to Regmi et al. (2014), mitochondrial degeneration may be related to age. Biopsies of human muscle reveal that characteristics such as decreased activities of tricarboxylic acid cycle enzymes and those related to oxidative phosphorylation and ATP synthesis may be related to factors such as mitochondrial volume decrease, increased oxidative stress, mitochondrial DNA (mtDNA), and/or altered mitochondrial morphology observed during aging of muscle cells (Bua et al., 2002; Marzetti et al., 2013). Valadão et al. (2017) also described similar ultrastructural changes in the mitochondria of the sternomastoid muscle of 12-months-old BACHD mice. In addition, several studies support the hypothesis that mitochondria dysfunction is involved in the pathophysiological process of several neurodegenerative diseases, particularly HD (Browne and Beal, 2004; Reddy et al., 2009).

In light of the morphological changes observed in the diaphragm NMJs of 12-months-old BACHD animals, we hypothesized that the neuromuscular function would also be affected in these mice. Therefore, we evaluated the overall safety margin of the synaptic transmission regarding the resistance to the neuromuscular blockade induced by pancuronium bromide or magnesium (del Castillo and Engbaek, 1954). No differences in the susceptibility of the neuromuscular blockade induced by pancuronium bromide or excess of magnesium were found between both genotypes, indicating that the safety margin of synaptic transmission was preserved in the diaphragm NMJs of BACHD mice. This suggests that the phrenic nerve of the diaphragm muscle of BACHD animals may be adapted to the period of exposure to HD or that the pre- or postsynaptic alterations were not enough to alter the neurotransmission process observed in our methodology.

Physiological tetanic stimulation induces depletion of ACh output, which is balanced by increased synthesis and transfer of neurotransmitter from mobilization stores (Padmaja and Mantha, 2002). However, the progressive increase of tetanic frequency can disrupt this balance and preparations with compromised safety margin will be more susceptible to such changes, presenting an absence of sustained tetanic stimulation, which is known as tetanus fade (Gissen and Katz, 1969). We used a tetanic protocol and evaluated the fatigue resistance of the phrenic-diaphragm preparation from BACHD and WT mice. Diaphragm muscle resistance to fatigue animals was not significantly different between the BACHD and WT mice. Importantly, the diaphragm muscle is vital for survival since it is involved in the respiratory process. Therefore, it is likely that even if morphological changes are present, the diaphragm probably has a higher resistance threshold than some other muscles when it undergoes functional tests. Future studies characterizing the contractile properties of other muscles may allow comparisons and provide insights on the mechanisms of diaphragm resistance.

\section{Conclusions}

In summary, here we provide evidence that aging maintains alterations in the diaphragm NMJs (i.e: reduced exocytosis and ACh release, and altered shape and size of synaptic vesicles) of 12months-old BACHD mice. Further, aging does not lead to further changes (i.e: in endocytosis, or muscle ultrastructure) in the BACHD mice diaphragm. Additionally, we noted that WT mice presented losses in the parameters analyzed, probably due to aging. This may have masked the effects of HD in the 12-months-old BACHD mice. Our functional analysis suggested that the phrenic nerve might be less affected by the neurodegeneration processes occurring in HD. Therefore, we propose that putative resistance mechanisms may be protecting this vital muscle to allow for survival. The results presented herein contribute to expand the current understanding of the effects of mHTT in the neuromuscular synapse and in the diaphragm muscle function.

\section{Conflicts of interest}

We have no conflict of interest to declare.

\section{Acknowledgements}

This work was supported by grants from FAPEMIG (\#00271-13), CNPq (\#467220/2014-0 and \#475735/2013-7), CAPES and IBROPROLAB 2017 (C.G). Cristina Guatimosim and Silvia Guatimosim are recipients of CNPq research fellowships. The authors would like to thank the Center for Acquisition and Processing of Images (CAPI/ ICB/UFMG) and the Microscopy Center at UFMG for providing the equipment and technical support for experiments involving tissue processing and electron microscopy.

\section{Appendix A. Supplementary data}

Supplementary data related to this article can be found at https://doi.org/10.1016/j.neuint.2018.03.007.

\section{References}

Aquilonius, S.M., Eckernås, S.A., Sundwall, A., 1975. Regional distribution of choline acetyltransferase in the human brain: changes in Huntington's chorea. J. Neurol. Neurosurg. Psychiatry 38, 669-677.

Baig, S.S., Strong, M., Quarrell, O.W., 2016. The global prevalence of Huntington's disease: a systematic review and discussion. Neurodegener. Dis. Manag. 6 (4), $331-343$.

Barstad, J.A., Lilleheil, G., 1968. Transversally cut diaphragm preparation from rat. An adjuvant tool in the study of the physiology and pharmacology of the myoneural junction. Arch. Int. Pharmacodyn. Ther. 175, 373-390.

Betz, W.J., Bewick, G.S., 1992. Optical analysis of synaptic vesicle recycling at the frog neuromuscular junction. Science 255, 200-203.

Betz, W.J., Mao, F., Smith, C.B., 1996. Imaging exocytosis and endocytosis. Curr. Opin. Neurobiol. 6 (3), 365-371.

Brotherton, A., Campos, L., Rowell, A., Zoia, V., Simpson, S.A., Rae, D., 2012. Nutritional management of individuals with Huntington's disease: nutritional guidelines. Neurodegener. Dis. Manag. 2 (1), 33-43.

Browne, S.E., Beal, M.F., 2004. The energetics of Huntington's disease. Neurochem. Res. 29 (3), 531-546.

Bua, E.A., McKiernan, S.H., Wanagat, J., McKenzie, D., Aiken, J.M., 2002. Mitochondrial abnormalities are more frequent in muscles undergoing sarcopenia. J. Appl. Physiol. 92, 2617-2624.

Budzinski, K.L., Allen, R.W., Fujimoto, B.S., Kensel-Hammes, P., Belnap, D.M., Bajjalieh, S.M., Chiu, D.T., 2009. Large structural change in isolated synaptic vesicles upon loading with neurotransmitter. Biophys. J. 97 (9), 2577-2584.

Caviston, J.P., Holzbaur, E.L.F., 2009. Caviston, J. P., Holzbaur, E. L. Huntingtin as an essential integrator of intracellular vesicular trafficking. Trends Cell Biol. 19 (4), $147-155$.

Chau, W.K., So, K.F., Tay, D., Dockery, P., 2000. A morphometric study of optic axons regenerated in a sciatic nerve graft of adult rats. Restor. Neurol. Neurosci. 16, $105-116$.

Croft, B.G., Fortin, G.D., Corera, A.T., Edwards, R.H., Beaudet, A., Trudeau, L.E. Fon, E.A., 2005. Normal biogenesis and cycling of empty synaptic vesicles in dopamine neurons of vesicular monoamine transporter 2 knockout mice. Mol. Biol. Cell 16, 306-315.

de Aragão, B.C., Rodrigues, H.A., Valadão, P.A.C., Camargo, W., Naves, L.A., Ribeiro, F.M., Guatimosim, C., 2016. Changes in structure and function of diaphragm neuromuscular junctions from BACHD mouse model for Huntington's disease. Neurochem. Int. 93, 64-72.

del Castillo, J., Engbaek, L., 1954. The nature of the neuromuscular block produced by magnesium. J. Physiol. 124, 370-384.

DiFiglia, M., Sapp, E., Chase, K., Schwarz, C., Meloni, A., Young, C., Reeves, S.A., 1995. Huntingtin is a cytoplasmic protein associated with vesicles in human and rat brain neurons. Neuron 14, 1075-1081.

Difiglia, M., Sapp, E., Chase, K.O., Davies, S.W., Bates, G.P., Vonsattel, J.P., Aronin, N., 
1997. Aggregation of huntingtin in neuronal intranuclear inclusions and dystrophic neurites in brain. Science 277 (5334), 1990-1993.

Elmqvist, D., Quastel, D.M., 1965. A quantitative study of end-plate potentials in isolated human muscle. J. Physiol. 178 (3), 505-529.

Farrar, A.M., Callahan, J.W., Abercrombie, E.D., 2011. Reduced striatal acetylcholine efflux in the R6/2 mouse model of Huntington's disease: an examination of the role of altered inhibitory and excitatory mechanisms. Exp. Neurol. 232, $119-125$.

Ferrante, R.J., Gutekunst, C.A., Persichetti, F., Mcneil, S.M., Kowall, N.W., Gusella, J.F., Macdonald, M.E., Beal, M.F., Hersch, S.M., 1997. Heterogeneous topographic and cellular distribution of huntingtin expression in the normal human neostriatum. J. Neurosci. 17, 3052-3063.

Finkbeiner, S., 2011. Huntington's disease. CSH. Perspect. Biol. 3 (6), a007476.

Gallacci, M., Oliveira, A.C., 1994. Pre-and postsynaptic mechanisms involved in tetanic fade induced by pancuronium in the isolated rat muscle. Pharmacol 49 (4), 265-270.

Gauthier, L.R., Charrin, B.C., Borrell-Pagès, M., Dompierre, J.P., Rangone, H., Cordelières, F.P., De Mey, J., MacDonald, M.E., Lessmann, V., Humbert, S., Saudou, F., 2004. Huntingtin controls neurotrophic support and survival of neurons by enhancing BDNF vesicular transport along microtubules. Cell 118 (1), 127-138.

Gissen, A.J., Katz, R.L., 1969. Twitch, tetanus and posttetanic potentiation as indices of nerve-muscle block in man. Anesthesiol 30 (5), 481-487.

Gray, M., Shirasaki, D.I., Cepeda, C., Andre, V.M., Wilburn, B., Lu, X.H., Yang, X.W., 2008. Full-length human mutant huntingtin with a stable polyglutamine repeat can elicit progressive and selective neuropathogenesis in BACHD mice. J. Neurosci. 28 (24), 6182-6195.

Harjes, P., Wanker, E.E., 2003. The hunt for huntingtin function: interaction partners tell many different stories. Trends Biochem. Sci. 28 (8), 425-433.

Heemskerk, A.W., Roos, R.A., 2011. Dysphagia in Huntington's disease: a review. Dysphagia 26 (1), 62-66.

Hoffner, G., Kahlem, P., Djian, P., 2002. Perinuclear localization of huntingtin as a consequence of its binding to microtubules through an interaction with $\beta-$ tubulin: relevance to Huntington's disease. J. Cell Sci. 115 (5), 941-948.

Imarisio, S., Carmichael, J., Korolchuk, V., Chen, C.W., Saiki, S., Rose, C., Krishna, G., Davies, J.E., Ttofi, E., Underwood, B.R., Rubinsztein, D.C., 2008. Huntington's disease: from pathology and genetics to potential therapies. Biochem. J. 412 (2), 191-209.

Jones, K., Pitceathly, R.D., Rose, M.R., McGowan, S., Hill, M., Badrising, U.A., Hughes, T., 2016. Interventions for dysphagia in long-term, progressive muscle disease. Cochrane Database Syst. Rev. 2, CD004303.

Khedraki, A., Reed, E.J., Romer, S.H., Wang, Q., Romine, W., Rich, M.M., Talmadge, R.J., Voss, A.A., 2017. Depressed synaptic transmission and reduced vesicle release sites in Huntington's disease neuromuscular junctions. J. Neurosci. 37 (34), 8077-8091.

Li, H., Wyman, T., Yu, Z.X., Li, S.H., Li, X.J., 2003. Abnormal association of mutant huntingtin with synaptic vesicles inhibits glutamate release. Hum. Mol. Genet. 12 (16), 2021-2030.

Li, S.H., Li, X.J., 2004. Huntingtin-protein interactions and the pathogenesis of Huntington's disease. Trends Genet. 20 (3), 146-154.

Marzetti, E., Calvani, R., Cesari, M., Buford, T.W., Lorenzi, M., Behnke, B.J., Leeuwenburgh, C., 2013. Mitochondrial dysfunction and sarcopenia of aging: from signaling pathways to clinical trials. Int. J. Biochem. Cell Biol. 45, 2288-2301.

Massouh, M., Wallman, M.J., Pourcher, E., Parent, A., 2008. The fate of the large striatal interneurons expressing calretinin in Huntington's disease. Neurosci. Res. 62 (4), 216-224.

McLachlan, E.M., Martin, A.R., 1981. Non-linear summation of end-plate potentials in the frog and mouse. J. Physiol. 311, 307-324.

McNeil, S.M., Novelletto, A., Srinidhi, J., Barnes, G., Kornbluth, I., Altherr, M.R., Myers, R.H., 1997. Reduced penetrance of the Huntington's disease mutation. Hum. Mol. Genet. 6 (5), 775-779.

Mielcarek, M., 2015. Huntington's disease is a multi-system disorder. Rare Dis. 3 (1), e1058464.

Morton, A.J., Edwardson, J.M., 2001. Progressive depletion of complexin II in a transgenic mouse model of Huntington's disease. J. Neurochem. 76 (1), $166-172$.

Padmaja, D., Mantha, S., 2002. Monitoring of neuromuscular junction. Indian J. Anaesth. 46 (4), 279-288.

Pratt, S.J., Shah, S.B., Ward, C.W., Inacio, M.P., Stains, J.P., Lovering, R.M., 2013. Effects of in vivo injury on the neuromuscular junction in healthy and dystrophic muscles. J. Physiol. 591, 559-570.

Reddy, P.H., Mao, P., Manczak, M., 2009. Mitochondrial structural and functional dynamics in Huntington's disease. Brain Res. Rev. 61 (1), 33-48.

Regmi, S.G., Rolland, S.G., Conradt, B., 2014. Age-dependent changes in mitochondrial morphology and volume are not predictors of lifespan. Aging (Albany NY) 6 (2), 118-130.

Ribchester, R.R., Thomson, D., Wood, N.I., Hinks, T., Gillingwater, T.H., Wishart, T.M. Morton, A.J., 2004. Progressive abnormalities in skeletal muscle and neuromuscular junctions of transgenic mice expressing the Huntington's disease mutation. Eur. J. Neurosci. 20, 3092-3114.

Rizzoli, S.O., Betz, W.J., 2004. The structural organization of the readily releasable pool of synaptic vesicles. Science 303 (5666), 2037-2039.

Rodrigues, H.A., Fonseca, M.D.C., Camargo, W.L., Lima, P.M.A., Martinelli, P.M., Naves, L.A., Guatimosim, C., 2013. Reduced expression of the vesicular acetylcholine transporter and neurotransmitter content affects synaptic vesicle distribution and shape in mouse neuromuscular junction. PLoS One 8 (11), e78342.

Roos, R.A., 2010. Huntington's disease: a clinical review. Orphanet J. Rare Dis. 5, 40-48.

Rozas, J.L., Gomez-Sanchez, L., Tomas-Zapico, C., Lucas, J.J., Fernandez-Chacon, R., 2011. Increased neurotransmitter release at the neuromuscular junction in a mouse model of polyglutamine disease. J. Neurosci. 31 (3), 1106-1113.

Rozas, J.L., Gómez-Sánchez, L., Tomás-Zapico, C., Lucas, J.J., Fernández-Chacón, R. 2010. Presynaptic dysfunction in Huntington's disease. Biochem. Soc. Trans. 38 (2), 488-492.

Rubinsztein, D.C., Leggo, J., Coles, R., Almqvist, E., Biancalana, V., Cassiman, J.J., Hayden, M.R., 1996. Phenotypic characterization of individuals with 30-40 CAC repeats in the Huntington disease (HD) gene reveals HD cases with 36 repeats and apparently normal elderly individuals with 36-39 repeats. Am. J. Hum. Genet. 59 (1), 16-22.

Sathasivam, K., Hobbs, C., Turmaine, M., Mangiarini, L., Mahal, A., Ber-taux, F. Wanker, E.E., Doherty, P., Davies, S.W., Bates, G.P., 1999. Formation of polyglutamine inclusions in non-CNS tissue. Hum. Mol. Genet. 8, 813-822.

Sari, Y., 2011. Huntington's disease: from mutant huntingtin protein to neurotrophic factor therapy. Int. J. Biomed. Sci. 7 (2), 89-100.

Sassone, J., Colciago, C., Cislaghi, G., Silani, V., Ciammola, A., 2009. Huntington's disease: the current state of research with peripheral tissues. Exp. Neurol. 219 (2), 385-397.

Saudou, F., Humbert, S., 2016. The biology of huntingtin. Neuron 89 (5), 910-926.

Smith, R., Brundin, P., Li, J.Y., 2005. Synaptic dysfunction in Huntington's disease: a new perspective. Cell. Mol. Life Sci. 62 (17), 1901-1912.

Smith, R., Chung, H., Rundquist, S., Maat-Schieman, M.L.C., Colgan, L., Englund, E., Li, J.Y., 2006. Cholinergic neuronal defect without cell loss in Huntington's disease. Hum. Mol. Genet. 15 (21), 3119-3131.

Smith, R., Klein, P., Koc-Schmitz, Y., Waldvogel, H.J., Faull, R.L.M., Brundin, P., Li, J.Y., 2007. Loss of SNAP- 25 and rabphilin 3a in sensory-motor cortex in Huntington's disease. J. Neurochem. 103 (1), 115-123.

Schulte, J., Troy Littleton, J., 2011. The biological function of the Huntingtin protein and its relevance to Huntington's Disease pathology. Curr. Trends Neurol. 1 (5), $65-78$.

The Huntington's Disease Collaborative Research Group., 1993.

Valadão, P.A.C., de Aragão, B.C., Andrade, J.N., Magalhães-Gomes, M.P.S. Foureaux, G., Joviano-Santos, J.V., Guatimosim, C., 2017. Muscle atrophy is associated with cervical spinal motoneuron loss in BACHD mouse model for Huntington's disease. Eur. J. Neurosci. 45 (6), 785-796.

Valdez, G., Tapia, J.C., Kang, H., Clemenson, G.D., Gage, F.H., Lichtman, J.W. Sanes, J.R., 2010. Attenuation of age- related changes in mouse neuromuscular synapses by caloric restriction and exercise. Proc. Natl. Acad. Sci. U. S. A 107 (33), 14863-14868.

Valdez, G., Tapia, J.C., Lichtman, J.W., Fox, M.A., Sanes, J.R., 2012. Shared resistance to aging and als in neuromuscular junctions of specific muscles. PLoS One 7 (4), e34640.

Van Der Burg, Jorien, M.M., Björkqvist, M., Brundin, P., 2009. Beyond the brain: widespread pathology in Huntington's disease. Lancet Neurol. 8, 765-774.

Van der Kloot, W., Molgo, J., Cameron, R., Colasante, C., 2002. Vesicle size and transmitter release at the frog neuromuscular junction when quantal acetylcholine content is increased or decreased. J. Physiol. 541, 385-393.

Vonsattel, G.J.P., DiFiglia, M., 1998. Huntington disease. J. Neuropathol. Exp. Neurol. 57 (5), 369-384.

Walker, F.O., 2007. Huntington's disease. Lancet 369 (9557), 218-228.

Wu, L.L., Zhou, X.F., 2009. Huntingtin associated protein 1 and its functions. Cell Adhes. Migrat. 3 (1), 71-76.

Yuen, E.Y., Wei, J., Zhong, P., Yan, Z., 2012. Disrupted GABAAR trafficking and synaptic inhibition in a mouse model of Huntington's disease. Neurobiol. Dis. 46 (2), 497-502.

Zielonka, D., Piotrowska, I., Marcinkowski, J.T., Mielcarek, M., 2014. Skeletal muscle pathology in Huntington's disease. Front. Physiol. 5, 380-385.

Zuccato, C., Valenza, M., Cattaneo, E., 2010. Molecular mechanisms and potential therapeutical targets in Huntington's disease. Physiol. Rev. 90 (3), 905-981. 